\title{
On development of fractional calculus during the last fifty years
}

\author{
J. A. Tenreiro Machado, Alexandra M. S. F. Galhano, Juan J. Trujillo
}

\section{Abstract}

Fractional calculus generalizes integer order derivatives and integrals. During the last half century a considerable progress took place in this scientific area. This paper addresses the evolution and establishes an assertive measure of the research development.

Keywords

Fractional calculus, Scientific evolution, Science metrics

\section{Introduction}

In 1695, in a letter to the French mathematician Guillaume de L'Ho pital, Gottfried Leibniz wrote "Can the meaning of derivatives with integer order be generalized to derivatives with non-integer orders?', L'Ho pital replied to Leibniz by formulating another question: "What if the order will be $\frac{1}{2}$ ?" Later, in another letter, Leibniz replied: "It will lead to a paradox, from which one day useful consequences will be drawn". The question posed by Leibniz for a fractional derivative became a topic for more than 300 years and many important mathematicians contributed to this theory over the years, namely Euler, Liouville, Fourier, Abel, Riemann, Weyl, just to name a few.

The calculation of derivatives or integrals of non-integer order is a branch of the classical calculus usually known as "Fractional Calculus" (FC). This term reflects the birth of the concept, but it is a misnomer and "integration and differentiation of arbitrary order" or "'generalized integro-differentiation", are more appropriate (Eames and Redheffer 1966; Machado et al. 2011). FC remained an abstract mathematical area and only in the last five 
decades researchers verified that fractional-order models capture intrinsically many characteristics of natural and artificial phenomena.

Bertram Ross (1974), shortly after his Ph.D. dissertation on FC, organized the First Conference on Fractional Calculus and its Applications at the University of New Haven in June 1974, and edited its proceedings. Keith B. Oldham and Jerome Spanier (1974) published the first monograph devoted to FC in 1974. Presently the series of books, journals and conferences devoted to FC and its applications includes many titles and this list is expected to grow more in the forthcoming years. Therefore, it is the right time to analyse the development that took place in this scientific area and to define some assertive measure of this evolution.

One of the metrics in science more well known is the so-called Moore's law that deals with the history of computing hardware (Moore 1965; Mollick 2006). A exponential trendline was proposed by Gordon E. Moore after noting that the number of components in integrated circuits was doubling every 2 years from the invention of the integrated circuit in 1958 until 1965. Therefore he predicted that the trend would continue "for at least ten years', which was proven to be accurate in the succeeding years. In fact, the trendline is used in the semiconductor industry for long-term planning and to define targets for research and development. However, recently the growth seems to be slowing down and transistor counts and densities is expected to double only every 3 years.

In scientific publications is often considered the Lotka's law (Machado et al. 2010b), named after Alfred J. Lotka. This trendline describes the frequency of publication by authors, noting that the number of authors making $n$ contributions is approximately $1 / n^{\mathrm{a}}$ of those making one contribution. It was observed that the power law trendline covers many fields and that a depends on the scientific area being reported values between 2 and 3 . In fact, Lotka's law is just a special case of the power law family of cases describing natural and man made phenomena (Dugowson 1994; Machado et al. 2013; Newman 2006]. Often the quantitative study of science focused on patterns in publications, such as, counting citations and co-authorship to measure impact and collaboration. However, science metrics offers the opportunity to understand scientific discovery and to influence the development of science (Arbesman 2011).

Probably one of the most well known speculation based on trendlines is due to Raymond Kurzweil (Machado et al. 2010a). He proposes a "technological singularity"' in the future and estimates that the humans species will be able to extend bodies and minds by means of technology. Kurzweil observes the exponential growth in the "law of accelerating returns"' and foresees what will occur in the future of mankind. Kurzweil's speculations and selective use of growth indices has been debated and vividly challenged. While this discussion is not the topic of the present paper it shows that the use of trendlines to describe the past is commonly accepted, but poses philosophical and conceptual dilemmas for forecasting the future. Bearing these ideas in mind, in the next section it is discussed the evolution in the area of FC. Some measures are proposed and the results analysed using histograms and trendlines.

\section{Fractional calculus: history and progress during the last decades}

FC emerged in 1695 in the sequel of the brilliant ideas of Gottfried Leibniz. Many relevant scientists supported these three centuries of progress in FC and Fig. 1 depicts a simple timeline. For general mathematics from $1000 \mathrm{AD}$ up to $1950 \mathrm{AD}$ we can mention the 1966 IBM poster, titled "Men of Modern Mathematics" (Carla et al. 2012), by Charles Eames 
and Raymond Redheffer (in 2012 IBM Corporation, with the assistance of the Eames Office, released an iPad application entitled "Minds of Modern Mathematics"' based on the poster and updated to the present), while for details in FC readers can get freely the posters entitled "Old History of Fractional Calculus" (Lotka 1926) and "Recent History of Fractional Calculus" (Machado 2011) in the Journal Fractional Calculus and Applied Analysis website.

The observation of these charts reveals intuitively that considerable progress took place and the question arises naturally if some quantification is possible. More precisely we are debating if some kind of measure of the scientific activity is possible, how to design it and, once we get some quantitative results, how to interpret them (Kurzweil 2005). The first assumption to formulate is that we will adopt a statistical procedure and that, for this purpose, significant volume of data is only available during the last 50 years. The second assumption is that we shall focus in publishing media that somehow reflect the existing

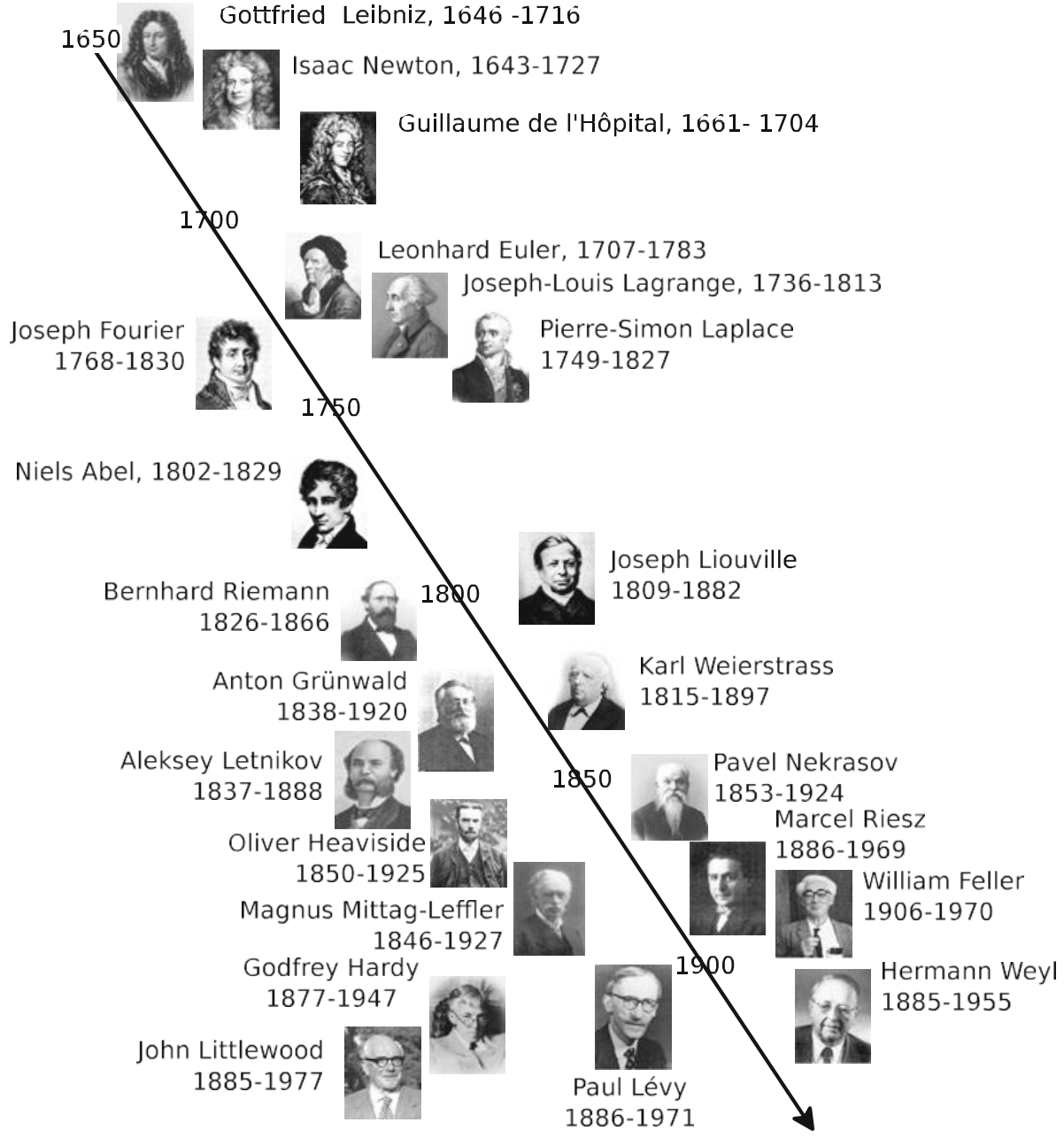

Fig. 1 Timeline of main scientists in the area of fractional calculus 
knowledge. This perspective corresponds to considering books and monographs as the physical objects to treat in a quantification procedure. No distinction will be made about language, authors, number of pages or publisher. Even so, two distinct approaches will be considered, namely books with author and books edited giving rise to distinct sets of measurements. This strategy entails pros and cons. While it provides an assertive "counting algorithm" it may fail to capture the whole scientific knowledge due to social or economical factors restricting access to such publications. Another possible set of measuring objects is represented by conferences in FC that emerged during the last decades. Nevertheless, it is difficult to distinguish such events in terms of number of participants and importance, since we have denominations such as special sessions, invited sections, workshops, conferences, or symposiums. Another possibility is to address published papers in journals, but again multiple difficulties arise ranging from defining the limits of what is FC, or not, and counting manuscripts in a plethora of journals from different publishers. Therefore, were considered the books with author and books edited published since 1966. The analysis is based on histograms constructed with $h=10$ years bins (i.e., 1966-1975 up to 1996-2005) with exception of the last 7 years period (i.e., 2006-2012). The value of books published per year $n_{i} / h_{i}$, where $n_{i}$ denotes the number of published objects during the period $h_{i} ; i 1 / 41 ; .$. .; 5 , is then plotted. Due to the scarcity of data the period 1966-1975 is not considered in the trendline calculation for the second index.

Figure 2 shows the histograms for the books with author and books edited indices. The exponential trendlines reveal a good correlation factor, but different growing rates. This discrepancy means that such indices describe only a part of the objects under analysis and common sense suggests that some value in between both cases is probably closer to the "true". These trend lines reflect the past and there is no guarantee that we can foresee the future as the Moore law seems to be demonstrating recently.

A similar conclusion can be drawn if we consider the cumulative values of books with author and books edited, that is, if we sum up the consecutive values. Plotting one variable

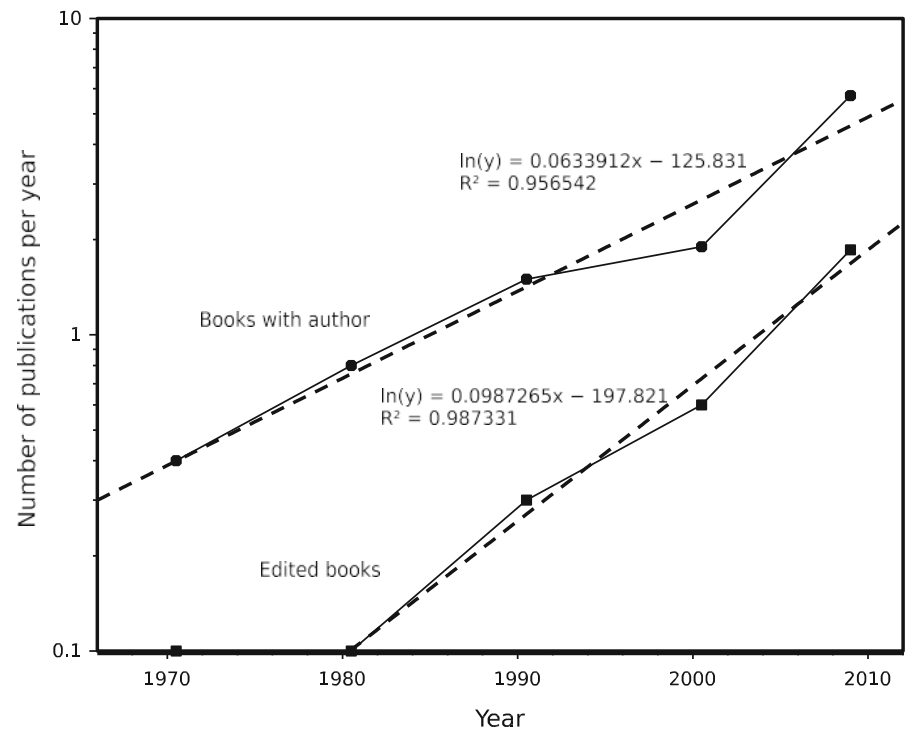

Fig. 2 Histograms and trendlines of the books with author and books edited published since 1966 in the area of fractional calculus 


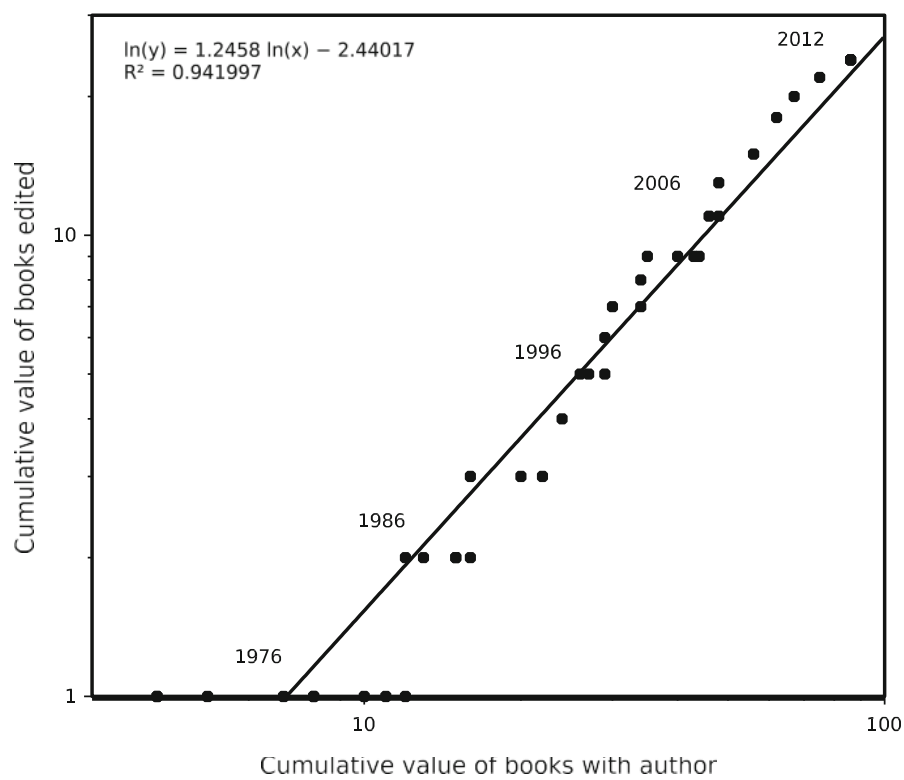

Fig. 3 Cumulative value of books with author versus cumulative value of books edited published since 1986 in the area of fractional calculus

against the other (having, therefore, time as a parametric variable) we get the chart depicted in Fig. 3. Due to the low number of observations in the initial decade, only the values from 1976 up to 2012 are represented. The power law trendline reveals again a steady evolution and that the books with author evolves slower than the books edited.

We believe that this study supports the intuitive knowledge of "progress", here measured by two distinct indices. In what concerns the different rates we consider them merely as a manifestation of the particular scientific area under analysis. It would be a relevant future work the comparison with other areas of active research in order to have a deeper understating of how the distinct indices captures the characteristics of each field.

Acknowledgements This work was supported, in part, by Government of Spain and FEDER Grant No. MTM2010-16499.

\section{References}

Arbesman, S. (2011). Quantifying the ease of scientific discovery. Scientometrics, 86(2), 245-250.

Carla, M. A., Pinto, A. M. L., \& Machado, J. A. T. (2012). A review of power laws in real life phenomena. Communications in Nonlinear Science and Numerical Simulation, 17(9), 3558-3578.

Dugowson, S. (1994). Les diffe'rentielles métaphysiques (histoire et philosophie de la généralisation de l'ordre de de rivation). PhD The`se, Universite' Paris Nord, Paris, France.

Eames, C., \& Redheffer, R. M., \& International Business Machines Corporation. (1966). Men of modern mathematics: A history chart of mathematicians from 1000 to 1900. Armonk: International Business Machines Corporation. Retrieved from http://books.google.pt/books?id=y8VaYgEACAAJ.

Kurzweil, R. (2005). The singularity Is near: When humans transcend biology. London: Viking Press. Lotka, A. J. (1926). The frequency distribution of scientific productivity. Journal of the Washington Academy of Sciences, 16(12), 317-324.

Machado, J. A. T. (2011). And i say to myself: “What a fractional world!’. Fractional Calculus \& Applied Analysis, 14(4), 635-654. 
Machado, J. A. T., Kiryakova, V., \& Mainardi, F. (2010a). A poster about the old history of fractional calculus. Fractional Calculus \& Applied Analysis, 13(4), 447-454.

Machado, J. A. T., Kiryakova, V., \& Mainardi, F. (2010b). A poster about the recent history of fractional calculus. Fractional Calculus \& Applied Analysis, 13(3), 329-334.

Machado, J. A. T., Kiryakova, V., \& Mainardi, F. (2011). Recent history of fractional calculus. Communications in Nonlinear Science and Numerical Simulations, 16(3), 1140-1153.

Machado, J. A. T, Galhano, A. M., \& Trujillo, J. J. (2013). Science metrics on fractional calculus development since 1966. Fractional Calculus \& Applied Analysis, 16(2), 479-500.

Mollick, E. (2006). Establishing Moore's law. IEEE Annals of the History of Computing, 28(3), 62-75.

Moore, G. E. (1965). Cramming more components onto integrated circuits. Electronics, 38(8), $114-117$.

Newman, M. E. J. (2006). Power laws, Pareto distributions and Zipf's law. Contemporary Physics, 46(5), 323-351.

Oldham, K. B., \& Spanier, J. (1974). The fractional calculus: Theory and application of differentiation and integration to arbitrary order. New York: Academic Press.

Ross, B. (ed.) (1974). Fractional calculus and its applications. In Proceedings of the International Conference, New Haven. New York: Springer-Verlag. 